\title{
EDITORIAL
}

\section{The Value of Learned Journals for Health Professionals}

\author{
Lindsay Edouard \\ Member of the Editorial Advisory Board, African Journal of Reproductive Health \\ Blue Bay, Mauritius
}

*For Correspondence: Email: soranae@gmail.com

As we celebrate the twenty-year anniversary of the African Journal of Reproductive Health (AJRH) this year ${ }^{1}$, it is apt to consider the changing role of learned journals over the last two decades specially with increasing access to the worldwide web. A muchneeded and welcome democratization in the availability of information has been accompanied regrettably by a concomitant overload from continual bombardment by email messages and postings from social media and at websites. As much of that information is not sieved, false news spread at an alarming rate with potentially detrimental effects on clinical consultations when misinformed patients query clinical decisions of health providers and demand inappropriate interventions specially drugs with supposedly miracle effects ${ }^{2}$. Whereas it is imperative to be selective in accessing information sources to avoid fake news, this approach is becoming difficult lately with the unfortunate official promotion of alternative facts in a post-truth context ${ }^{3}$. With triage becoming increasingly difficult, there is renewed interest in professional journals and textbooks which are now aimed at not only students but also health planners and development practitioners ${ }^{4}$.

For journal contents, there is the delicate 'town and gown' balance between service provision and academia but that gap is closing with the implementation of evidence-based care. Health journals that developed from broadsheets and newsletters of professional associations ${ }^{5}$ justifiably continue their role of mouthpiece by including items such as minutes of annual general meeting, reports from committees and administrative notices. Through ownership of a learned journal, a professional association demonstrates that it is not merely a trade union but it is also desirable for the journal editor to be given full independence free from pressure regarding both contents, specially as pertaining to standpoints of the professional association, and financial constraints necessitating advertising revenue. During the first decade of the African Journal of Reproductive Health, three leading medical journals in North America had to revoke their editors over editorial independence ${ }^{6}$.
Peer review of manuscripts is an integral part of the editorial process to ensure the quality of articles published in a learned journal. Whereas guidelines exist, problems can arise in their implementation ${ }^{7}$. Having the final word, the editor should be impartial, abandon personal interests and neither give-in to pressure groups nor favor friends: a constraining situation similar to that faced by the Speaker in a Westminster-style parliament ${ }^{8}$.

For service provider, policy maker or planner, it is much easier to shoot from the hip by taking decisions spontaneously on the spot as opposed to consulting the health literature for up-to-date evidence. With the electronic age, the vade-mecum in the pocket of the white coat of the clinician has often been replaced by bookmarks on personal digital devices to access reliable websites for official guidelines and online journals. Unfortunately, a substantial portion of the health literature is accessible only upon payment thereby making it largely inaccessible in developing countries where practitioners are those most in need.

The decision-making process, whether at the bedside or policy desk, should ideally draw upon evidence specially from research findings ${ }^{9,10}$. Journal articles should clearly state their contribution to the existing body of knowledge. Therefore, the publication of research articles is part of a two-way process: reporting of findings to supplement existing systematic reviews which must be used to formulate subsequent research projects that will uncover new facts ${ }^{11}$. With the dearth of pertinent findings from low and middleincome countries, this issue is crucial to generate the information database for their implementation of evidence-based practices.

With the current trend towards quantification of all issues, metrics are assuming more importance in assessing journals through proxy indices, such as impact factor, which have their own limitations. It should not be forgotten that, in the first place, a journal should meet the specific needs of its target readership whereas prominence on the worldwide web is desirable for generating awareness of journal contents during 
literature searches.

Having its origins within a research centre, the African Journal of Reproductive Health has had an exemplary path as pertaining to editorial independence, quarterly periodicity of publication and quality of articles besides availability of issues at a dedicated website and inclusion in PubMed from the very beginning. Having been recognized as the leading regional journal in its field, the challenge now lies in consolidating its multidisciplinary approach, with a wide perspective on reproductive health, to promote its utilization for policy formulation in the quest for achieving the health-related sustainable development goals.

\section{Conflict of Interest}

None

\section{References}

1. Okonofua F and Omonkhua A. African Journal of Reproductive Health at 20: Looking Back and Looking Forward. Afr J Reprod Health 2017; 21:11-13.
2. Robledo I and Jankovic J. Media hype: patient and scientific perspectives on misleading medical news. Mov Disord 2017; doi: $10.1002 / \mathrm{mds} .26993$.

3. Editorial. Free speech and facts in the Trump era. Lancet 2017; 389: 478.

4. Okonofua FE. (Ed.) Confronting the challenge of reproductive health in Africa: a textbook for students and development practitioners. Boca Raton, Florida: Brown Walker, 2014.

5. Edouard L. International collaboration for sexual and reproductive health. J Fam Plann Reprod Health Care 2016; 42: 298-300.

6. Van Der Weyden MB. On being the Editor of the Medical Journal of Australia: living dangerously. Mens Sana Monogr 2012; 10: 150-157.

7. Homedes $\mathrm{N}$ and Ugalde $\mathrm{A}$. Are private interests clouding the peer-review process of the WHO Bulletin? A case study. Account Res 2016; 23: 309-317

8. King HM. The Speaker and parliament. Birmingham: Stacey, 1973: 22, 25, 27.

9. Pisani E. The wisdom of whores: bureaucrats, brothels and the business of AIDS. Toronto: Viking, 2008:188-226

10. Bullough VL. Science in the bedroom: a history of sex research. New York, NY: Harper Collins, 1994.

11. Chalmers I and Fox DM. Increasing the incidence and influence $f$ systematic reviews on health policy and practice. Am J Public Health 2016; 106: 11-13 


\title{
EDITORIAUX
}

\section{La Valeur des Revues Scolaires pour les Professionnels de la Santé}

\author{
Lindsay Edouard
}

Membre du Conseil consultatif de rédaction, Blue Bay, Maurice

*Pour la correspondance: Courriel: soranae@gmail.com

Alors que nous célébrons le vingtième anniversaire de la African Journal of Reproductive Health $(A J R H)$ cette année ${ }^{1}$, il est approprié de considérer le rôle changeant des revues scolaires au cours des deux dernières décennies, surtout avec l'accès croissant au web mondial. Une démocratisation très nécessaire et bienvenue dans la disponibilité de l'information a été accompagnée, malheureusement, d'une surcharge concomitante du bombardement continu par courriels et publications des réseaux sociaux et des sites Web. La plupart de ces informations ne sont pas tamisées, de fausses nouvelles se propagent à un rythme alarmant avec des effets potentiellement néfastes sur les consultations cliniques lorsque des patients mal informés mettent en question les décisions cliniques des fournisseurs de soins de santé et demandent des interventions inappropriées en particulier des drogues ayant des effets soidisant miracles ${ }^{2}$. Alors qu'il est impératif d'être sélectif dans l'accès aux sources d'information pour éviter de fausses nouvelles, cette approche devient difficile récemment avec la mauvaise promotion officielle de faits alternatifs dans un contexte post-vérité ${ }^{3}$. Si le triage devient de plus en plus difficile, il y a un intérêt renouvelé pour les revues professionnelles et les manuels scolaires qui visent maintenant non seulement les étudiants, mais aussi les planificateurs de la santé et les praticiens du développement ${ }^{4}$.

Pour le contenu de la revue, il y a l'équilibre délicat de la «ville et de la robe» entre la prestation des services et le milieu universitaire, mais cette lacune se reduit par la mise en place de soins fondés sur des données probantes. Les revues de santé qui ont été élaborées à partir de feuilles de bord et des bulletins d'information d'associations professionnelles ${ }^{5}$ continuent à juste titre à jouer leur rôle de porte-parole en incluant des articles tels que les procès-verbaux de l'assemblée générale annuelle, les rapports des comités et les avis administratifs. Grâce à la propriété d'une revue scolaire, une association professionnelle démontre que ce n'est pas simplement un syndicat, mais il est également souhaitable que le rédacteur de revue bénéficie d'une indépendance totale sans pression sur les deux contenus, surtout en ce qui concerne les points de vue de l'association professionnelle, et les contraintes financières nécessitant des revenus publicitaires. Au cours de la première décennie de 1'African Journal of Reproductive Health, trois revues médicales de premier plan en Amérique du Nord ont dû révoquer leurs rédacteurs à l'égard de l'indépendance éditoriale ${ }^{6}$.

L'examen des manuscrits par les pairs fait partie intégrante du processus éditorial pour assurer la qualité des articles publiés dans une revue. Alors que des lignes directrices existent, des problèmes peuvent survenir dans leur mise en œuvre $^{7}$. Ayant le dernier mot, le rédacteur devrait être impartial, abandonner les intérêts personnels et ne pas céder aux groupes de pression ni favoriser les amis: une situation contraignante semblable à celle rencontrée par le Président de la Chambre des commune dans un parlement de style Westminster ${ }^{8}$.

Pour le fournisseur de services, le décideur ou le planificateur, il est beaucoup plus facile de tirer de la hanche en prenant des décisions spontanément sur place, par opposition à la consultation de la documentation sur la santé pour des preuves mises à jour. Avec l'âge électronique, le vade-mecum dans la poche de la blouse blanche 
du clinicien a souvent été remplacé par des signets sur des dispositifs numériques personnels pour accéder à des sites Web fiables pour les directives officielles et les revues en ligne. Malheureusement, une partie importante de la documentation sur la santé n'est accessible qu'avec le paiement, ce qui la rend largement inaccessible dans les pays en développement où les praticiens sont les plus démunis.

Le processus de prise de décision, qu'il s'agisse du bureau de chevet ou de la politique, devrait idéalement s'appuyer sur des preuves spécialement tirées des résultats de recherche ${ }^{9,10}$. Les articles de revue devraient clairement indiquer leur contribution à l'ensemble des connaissances existantes. Par conséquent, la publication d'articles de recherche fait partie d'un processus à double sens: le rapport des résultats pour compléter les examens systématiques existants qui doivent être utilisés pour formuler des projets de recherche ultérieurs qui découvriront de nouveaux faits ${ }^{1}$. Compte tenu de la pénurie de résultats pertinents des pays à revenu faible et intermédiaire, cette question est cruciale pour générer la base de données d'information pour la mise en œuvre de pratiques fondées sur des données probantes.

Etant donné la tendance actuelle à la quantification de tous les problèmes, les indicateurs prennent plus d'importance dans l'évaluation des revues par des indices de proxy, tels que les facteurs d'impact, qui ont leurs propres limites. Il ne faut pas oublier que, d'une part, une revue devrait répondre aux besoins spécifiques de ses lecteurs cibles, alors que la notoriété sur le Web mondial est souhaitable pour générer une prise de conscience du contenu de la revue pendant les recherches dans la documentation.

Ayant son origine d'un centre de recherche, la revue Journal Africain of Reproductive Health a eu un cheminement exemplaire en ce qui concerne l'indépendance éditoriale, la périodicité trimestrielle de la publication et la qualité des articles à part la disponibilité des problèmes sur un site dédié et l'inclusion dans PubMed dès le début.
Ayant été reconnu comme le premier revue régionale dans son domaine, le défi réside maintenant dans la consolidation de son approche pluridisciplinaire, avec une large perspective sur la santé de la reproduction, pour promouvoir son utilisation pour la formulation des politiques dans la quête de la réalisation des objectifs de développement durable liés à la santé.

\section{Conflit d'intérêts}

Aucune

\section{Références}

1. Okonofua F et Omonkhua A. African Journal of Reproductive Health à 20 ans: Regarder en arrière et regarder vers l'avant. Afr J Reprod Health 2017; 21: 11-13.

2. Robledo I et Jankovic J. Battage publicitaire sur le media: perspectives des patients et scientifiques sur les nouvelles médicales trompeuses. Mov Disord 2017; Doi: 10.1002 / mds.26993.

3. Éditorial. La liberté de parole et les faits dans l'ère de Trump. Lancet 2017; 389: 478

4. Okonofua FE. (Ed.) Relever le défi de santé de la reproduction en Afrique: un manuel pour les étudiants et les praticiens du développement. Boca Raton, Floride: Brown Walker, 2014.

5. Edouard L. Collaboration internationale pour la santé sexuelle et reproductive. J Fam Plann Reprod Health Care 2016; 42: 298-300.

6. Van Der Weyden MB. En tant que le rédacteur en chef du Medical Journal of Australia: vivre dangereusement. Mens Sana Monogr 2012; 10: 150-157.

7. Homedes $\mathrm{N}$ et Ugalde A. Les intérêts privés nuisent-ils au processus d'examen par les pairs du Bulletin de l'OMS? Une étude de cas. Account Res 2016; 23: 309-317.

8. King HM. Le président et le parlement. Birmingham: Stacey, 1973: 22, 25, 27.

9. Pisani E. La sagesse des prostituées: les bureaucrates, les maisons closes et les affaires du sida. Toronto: Viking, 2008: 188- 226.

10. Bullough VL. La science dans la chambre: une histoire de recherche sur le sexe. New York, NY: Harper Collins, 1994.

11. Chalmers I et Fox DM. Accroître l'incidence et l'influence des examens systématiques sur les politiques et les pratiques en matière de santé Am J Public Health 2016; 106: 11-13. 